\title{
On the Strong Law of Large Numbers for Sequences of Pairwise Independent Random Variables
}

\author{
Valery Korchevsky*
}

\begin{abstract}
We establish new sufficient conditions for the applicability of the strong law of large numbers (SLLN) for sequences of pairwise independent non-identically distributed random variables. These results generalize Etemadi's extension of Kolmogorov's SLLN for identically distributed random variables. Some of the obtained results hold with an arbitrary norming sequence in place of the classical normalization.
\end{abstract}

Keywords: strong law of large numbers, pairwise independent random variables.

\section{Introduction}

Let $\left\{X_{n}\right\}_{n=1}^{\infty}$ be a sequence of random variables defined on the same probability space and put $S_{n}=\sum_{k=1}^{n} X_{k}$.

The classical Kolmogorov's theorem states that if $\left\{X_{n}\right\}_{n=1}^{\infty}$ is a sequence of independent identically distributed random variables and $E\left|X_{1}\right|<\infty$ then $S_{n} / n \rightarrow E X_{1}$ almost surely. Etemadi 4 generalized the Kolmogorov theorem replacing the mutual independence assumption by the pairwise independence assumption.

Theorem A (4]). Let $\left\{X_{n}\right\}_{n=1}^{\infty}$ be a sequence of pairwise independent identically distributed random variables. If $E\left|X_{1}\right|<\infty$ then $S_{n} / n \rightarrow E X_{1}$ almost surely.

One can find further extensions of the Kolmogorov theorem to wide classes of dependent random variables in the papers [6] and [7].

In the present work we generalize Theorem $\mathrm{A}$ to non-identically distributed random variables. This problem was considered in the several papers. Chandra and Goswami $[3]$ established the following result.

Theorem B ([3]). Let $\left\{X_{n}\right\}_{n=1}^{\infty}$ be a sequence of pairwise independent random variables and put $G(x)=\sup _{n \geq 1} P\left(\left|X_{n}\right|>x\right)$ for $x \geq 0$. If

$$
\int_{0}^{\infty} G(x) d x<\infty
$$

\footnotetext{
*Saint-Petersburg State University of Aerospace Instrumentation, Saint-Petersburg.

E-mail: valery.korchevsky@gmail.com
} 
Then

$$
\frac{1}{n} \sum_{k=1}^{n} c_{k}\left(X_{k}-E X_{k}\right) \rightarrow 0 \quad \text { a.s. } \quad(n \rightarrow \infty)
$$

for each bounded sequence $\left\{c_{n}\right\}$.

The following result was obtained by Bose and Chandra [1].

Theorem C ([1]). Let $\left\{X_{n}\right\}_{n=1}^{\infty}$ be a sequence of pairwise independent random variables. Suppose that

$$
\begin{gathered}
\int_{0}^{\infty} G(x) d x<\infty \quad \text { with } G(x)=\sup _{n \geq 1} \frac{1}{n} \sum_{k=1}^{n} P\left(\left|X_{k}\right|>x\right) \quad \text { for all } x \geq 0, \\
\sum_{n=1}^{\infty} P\left(\left|X_{n}\right|>n\right)<\infty
\end{gathered}
$$

Then

$$
\frac{S_{n}-E S_{n}}{n} \rightarrow 0 \quad \text { a.s. }
$$

Kruglov [5] proved the next generalization of Theorem A.

Theorem D ([5]). Let $\left\{X_{n}\right\}_{n=1}^{\infty}$ be a sequence of pairwise independent random variables. Assume that

$$
\sup _{n \geq 1} E\left|X_{n}\right|<\infty .
$$

If there exists a random variable $X$ such that $E|X|<\infty$ and

$$
\sup _{n \geq 1} \frac{1}{n} \sum_{k=1}^{n} P\left(\left|X_{k}\right|>x\right) \leq C P(|X|>x) \quad \text { for all } x \geq 0,
$$

where $C$ is a positive constant, then relation (2) holds.

The aim of present work is to generalize Theorems $\mathrm{C}$ and $\mathrm{D}$, We present a generalization of Theorem $\mathrm{C}$ using an arbitrary norming sequence in place of the classical normalization. Furthermore we show that condition (3) in Theorems D can be dropped.

In order to prove the theorems in the present work, we use methods developed by Bose and Chandra [1] (see also Chandra 2]).

\section{Main results}

Theorem 1. Let $\left\{X_{n}\right\}_{n=1}^{\infty}$ be a sequence of pairwise independent random variables. Assume that $\left\{a_{n}\right\}_{n=1}^{\infty}$ is non-decreasing unbounded sequence of positive numbers. Suppose that

$$
\int_{0}^{\infty} G(x) d x<\infty \quad \text { with } G(x)=\sup _{n \geq 1} \frac{1}{a_{n}} \sum_{k=1}^{n} P\left(\left|X_{k}\right|>x\right) \quad \text { for all } x \geq 0,
$$




$$
\sum_{n=1}^{\infty} P\left(\left|X_{n}\right|>a_{n}\right)<\infty
$$

Then

$$
\frac{S_{n}-E S_{n}}{a_{n}} \rightarrow 0 \quad \text { a.s. }
$$

Theorem [1 generalizes Theorem $\left[\right.$ ] which corresponds to the case $a_{n}=n$ for all $n \geq 1$.

Theorem 2. Let $\left\{X_{n}\right\}_{n=1}^{\infty}$ be a sequence of pairwise independent random variables. If there exists function $H(x)$ such that $H(x)$ is non-increasing in the interval $x \geq 0$,

$$
\int_{0}^{\infty} H(x) d x<\infty, \quad \text { and } \quad \sup _{n \geq 1} \frac{1}{n} \sum_{k=1}^{n} P\left(\left|X_{k}\right|>x\right) \leq H(x) \quad \text { for all } x \geq 0,
$$

then

$$
\frac{S_{n}-E S_{n}}{n} \rightarrow 0 \quad \text { a.s. }
$$

As a consequence of Theorem 2 we immediately obtain the following result.

Corollary 1. Let $\left\{X_{n}\right\}_{n=1}^{\infty}$ be a sequence of pairwise independent random variables. If there exists a random variable $X$ such that $E|X|<\infty$ and

$$
\sup _{n \geq 1} \frac{1}{n} \sum_{k=1}^{n} P\left(\left|X_{k}\right|>x\right) \leq C P(|X|>x) \quad \text { for all } x \geq 0,
$$

where $C$ is a positive constant, then

$$
\frac{S_{n}-E S_{n}}{n} \rightarrow 0 \quad \text { a.s. }
$$

Corollary 1 shows that we can omit condition (3) in Theorem D

\section{Proofs}

To prove Theorems 1 we need the following proposition that is a consequence of Theorem 1 in $[3]$.

Lemma 1. Let $\left\{X_{n}\right\}_{n=1}^{\infty}$ be a sequence of non-negative random variables with finite variances. Assume that $\left\{a_{n}\right\}_{n=1}^{\infty}$ is non-decreasing unbounded sequence of positive numbers. Suppose that

$$
\operatorname{Var}\left(S_{n}\right) \leq C \sum_{k=1}^{n} \operatorname{Var}\left(X_{k}\right) \quad \text { for all } n \geq 1,
$$

where $C$ is a positive constant,

$$
\sum_{n=1}^{\infty} \frac{\operatorname{Var}\left(X_{n}\right)}{a_{n}^{2}}<\infty
$$




$$
\sup _{n \geq 1} \frac{1}{a_{n}} \sum_{k=1}^{n} E X_{n}<\infty .
$$

Then

$$
\frac{S_{n}-E S_{n}}{a_{n}} \rightarrow 0 \quad \text { a.s. }
$$

Proof of Theorem [1. Note that for pairwise independent random variables $X_{n}$, the positive parts $X_{n}^{+}:=\max \left\{0, X_{n}\right\}$ are pairwise independent. Likewise, the $X_{n}^{-}:=\max \left\{0,-X_{n}\right\}$ are pairwise independent. Thus it is enough to prove the theorem separately for the positive and negative parts. So we can assume that $X_{n} \geq 0$ for all $n \geq 1$.

Let $Y_{n}=X_{n} \mathbb{I}_{\left\{X_{n} \leq a_{n}\right\}}, T_{n}=\sum_{k=1}^{n} Y_{k}$ for every $n \geq 1$. To prove the theorem, it is sufficient to show that

$$
\begin{gathered}
\frac{E S_{n}-E T_{n}}{a_{n}} \rightarrow 0 \quad(n \rightarrow \infty), \\
\frac{T_{n}-E T_{n}}{a_{n}} \rightarrow 0 \quad \text { a.s., } \\
\frac{S_{n}-T_{n}}{a_{n}} \rightarrow 0 \quad \text { a.s. }
\end{gathered}
$$

Note that for any non-negative random variable $Z$ and $a>0$

$$
E\left(Z \mathbb{I}_{\{Z>a\}}\right)=a P(Z>a)+\int_{a}^{\infty} P(Z>x) d x
$$

and

$$
E\left(Z \mathbb{I}_{\{Z \leq a\}}\right) \leq \int_{0}^{a} P(Z>x) d x
$$

Fix an integer $N \geq 1$. Then, using (4) and (12), for $n>N$ we obtain

$$
\begin{aligned}
& E S_{n}-E T_{n}=\sum_{k=1}^{n} E\left(X_{k} \mathbb{I}_{\left\{X_{k}>a_{k}\right\}}\right) \\
& =\sum_{k=1}^{n} a_{k} P\left(X_{k}>a_{k}\right)+\sum_{k=1}^{n} \int_{a_{k}}^{\infty} P\left(X_{k}>x\right) d x \\
& =\sum_{k=1}^{n} a_{k} P\left(X_{k}>a_{k}\right)+\sum_{k=1}^{N} \int_{a_{k}}^{\infty} P\left(X_{k}>x\right) d x+\sum_{k=N+1}^{n} \int_{a_{k}}^{\infty} P\left(X_{k}>x\right) d x \\
& \leq \sum_{k=1}^{n} a_{k} P\left(X_{k}>a_{k}\right)+\sum_{k=1}^{N} \int_{0}^{\infty} P\left(X_{k}>x\right) d x+\sum_{k=1}^{n} \int_{a_{N}}^{\infty} P\left(X_{k}>x\right) d x \\
& \leq \sum_{k=1}^{n} a_{k} P\left(X_{k}>a_{k}\right)+a_{N} \int_{0}^{\infty} G(x) d x+a_{n} \int_{a_{N}}^{\infty} G(x) d x .
\end{aligned}
$$


Condition (51) and Kronecker's lemma (see, for example, [8]) imply that

$$
\frac{1}{a_{n}} \sum_{k=1}^{n} a_{k} P\left(X_{k}>a_{k}\right) \rightarrow 0 \quad(n \rightarrow \infty) .
$$

Thus for each $N \geq 1$ we have

$$
\limsup _{n \rightarrow \infty} \frac{1}{a_{n}}\left(E S_{n}-E T_{n}\right) \leq \int_{a_{N}}^{\infty} G(x) d x
$$

so we get assertion (9) by letting $N \rightarrow \infty$.

To establish (10) we shall prove that conditions of Lemma 1 are satisfied for sequence $\left\{Y_{n}\right\}_{n=1}^{\infty}$. It follows from pairwise independence of random variables $Y_{n}$ that assertion (7) is satisfied for sequence $\left\{Y_{n}\right\}_{n=1}^{\infty}$.

Using (41) and (13), we obtain

$$
\begin{aligned}
\sum_{n=1}^{\infty} \frac{\operatorname{Var}\left(Y_{n}\right)}{a_{n}^{2}} \leq \sum_{n=1}^{\infty} \frac{E\left(X_{n}^{2} \mathbb{I}_{\left\{X_{n} \leq a_{n}\right\}}\right)}{a_{n}^{2}} \leq \sum_{n=1}^{\infty} \frac{1}{a_{n}^{2}} \int_{0}^{a_{n}^{2}} P\left(X_{n}>x^{1 / 2}\right) d x \\
\leq 2 \sum_{n=1}^{\infty} \frac{1}{a_{n}^{2}} \int_{0}^{a_{n}} y P\left(X_{n}>y\right) d y \leq 4 \sum_{n=1}^{\infty} \frac{1}{\left(\left[a_{n}\right]+1\right)^{2}} \int_{0}^{\left[a_{n}\right]+1} y P\left(X_{n}>y\right) d y \\
\leq 8 \sum_{n=1}^{\infty} \sum_{j=\left[a_{n}\right]+1}^{\infty} \frac{1}{j^{3}} \sum_{k=1}^{\left[a_{n}\right]+1} \int_{k-1}^{k} y P\left(X_{n}>y\right) d y \\
\leq 8 \sum_{n=1}^{\infty} \sum_{j=\left[a_{n}\right]+1}^{\infty} \frac{1}{j^{3}} \sum_{k=1}^{j} \int_{k-1}^{k} y P\left(X_{n}>y\right) d y \\
\leq 8 \sum_{j=\left[a_{1}\right]+1}^{\infty} \sum_{n: a_{n} \leq j} \frac{1}{j^{3}} \sum_{k=1}^{j} \int_{k-1}^{k} y P\left(X_{n}>y\right) d y \\
\leq 8 \sum_{j=1}^{\infty} \sum_{k=1}^{j} \frac{1}{j^{2}} \int_{k-1}^{k} y \frac{\sum_{n: a_{n} \leq j} P\left(X_{n}>y\right)}{j} d y \\
\leq 8 \sum_{j=1}^{\infty} \sum_{k=1}^{j} \frac{1}{j^{2}} \int_{k-1}^{k} y G(y) d y=8 \sum_{k=1}^{\infty} \sum_{j=k}^{\infty} \frac{1}{j^{2}} \int_{k-1}^{k} y G(y) d y \\
\leq 16 \sum_{k=1}^{\infty} \frac{1}{k} \int_{k-1}^{k} y G(y) d y \leq 16 \sum_{k=1}^{\infty} \int_{k-1}^{k} G(y) d y=16 \int_{0}^{\infty} G(y) d y<\infty
\end{aligned}
$$

where $\left[a_{n}\right]$ is the integer part of $a_{n}$. Hence condition (8) is satisfied for sequence $\left\{Y_{n}\right\}_{n=1}^{\infty}$.

For each $n \geq 1$ we have

$$
\frac{1}{a_{n}} \sum_{k=1}^{n} E Y_{k} \leq \frac{1}{a_{n}} \sum_{k=1}^{n} E X_{k}=\frac{1}{a_{n}} \sum_{k=1}^{n} \int_{0}^{\infty} P\left(X_{k}>x\right) d x \leq \int_{0}^{\infty} G(x) d x<\infty .
$$


Thus the sequence of random variables $\left\{Y_{n}\right\}_{n=1}^{\infty}$ satisfies conditions of Lemma 1] so relation (10) holds.

To complete the proof it remains to verify assertion (11). Using (5), we obtain

$$
\sum_{n=1}^{\infty} P\left(X_{n} \neq Y_{n}\right)=\sum_{n=1}^{\infty} P\left(X_{n}>a_{n}\right)<\infty
$$

From Borel-Cantelli lemma and relation (10) it follows that

$$
\frac{S_{n}-E T_{n}}{a_{n}} \rightarrow 0 \quad \text { a.s. }
$$

Thus (11) follows from (10) and (14).

Proof of Theorem Q . As $H(x)$ is non-increasing in the interval $x \geq 0$, condition $\int_{0}^{\infty} H(x) d x<$ $\infty$ implies that $\sum_{k=0}^{\infty} 2^{k} H\left(2^{k}\right)<\infty$. Thus using (6) we have

$$
\begin{aligned}
\sum_{n=2}^{\infty} P\left(\left|X_{n}\right|\right. & >n)=\sum_{k=0}^{\infty} \sum_{n=2^{k}+1}^{2^{k+1}} P\left(\left|X_{n}\right|>n\right) \\
& \leq \sum_{k=0}^{\infty} \sum_{n=2^{k}+1}^{2^{k+1}} P\left(\left|X_{n}\right|>2^{k}\right) \leq \sum_{k=0}^{\infty} \sum_{n=1}^{2^{k+1}} P\left(\left|X_{n}\right|>2^{k}\right) \leq \sum_{k=0}^{\infty} 2^{k+1} H\left(2^{k}\right)<\infty
\end{aligned}
$$

Now, taking into account that (6) implies (1), we can conclude that the desired result follows from Theorem C]

\section{References}

[1] Bose A., Chandra T.K. A note on the strong law of large numbers. Calcutta Statistical Association Bulletin 44, 115-122 (1994)

[2] Chandra T.K. Laws of large numbers. Narosa Publishing House. New Delhi (2012)

[3] Chandra T.K., Goswami A. Cesáro uniform integrability and a strong laws of large numbers. Sankhyā, Ser. A 54, 215-231 (1992)

[4] Etemadi N. An elementary proof of the strong law of large numbers. Z. Wahrscheinlichkeitstheorie verw. Geb. 55, 119-122 (1981)

[5] Kruglov V.M. Strong law of large numbers. Stability Problems for Stochastic Models (Zolotarev V.M., Kruglov V.M., Korolev V.Yu., eds.) TVP/VSP. Moscow-Utrecht, 139150 (1994)

[6] Matula $P$. A note on the almost sure convergence of sums negatively dependent random variables. Statist. Probab. Lett. 15, 209-213 (1992) 
[7] Matula P. On some families of AQSI random variables and related strong law of large numbers. Appl. Math. E-Notes, 5, 31-35 (2005)

[8] Petrov V.V. Limit theorems of probability theory. Clarendon Press. Oxford (1995) 